Supporting Information for:

\title{
Iron tricarbonyl stabilized pentadienyl cation as initiator for cascade polycyclizations; a diastereoselective entry into octahydrophenanthrenes.
}

\author{
Anthony J. Pearson* and Victor P. Ghidu \\ Case Western Reserve University, Department of Chemistry, \\ Cleveland 44106, $\mathrm{OH}$. \\ anthony.pearson@case.edu
}

General procedures

${ }^{1} \mathrm{H},{ }^{13} \mathrm{C}$ spectra, Compound $\mathrm{lb}$

${ }^{1} \mathrm{H},{ }^{13} \mathrm{C}$ spectra, Compound $17 \mathrm{a}$

${ }^{1} \mathrm{H},{ }^{13} \mathrm{C}$ spectra, Compound $17 \mathrm{~b}$

${ }^{1} \mathrm{H},{ }^{13} \mathrm{C}$ spectra, Compound $17 \mathrm{c}$

${ }^{1} \mathrm{H},{ }^{13} \mathrm{C}$ spectra, Compound $17 \mathrm{~d}$

${ }^{1} \mathrm{H},{ }^{13} \mathrm{C}$ spectra, Compound $18 \mathrm{a}$

${ }^{1} \mathrm{H},{ }^{13} \mathrm{C}$ spectra, Compound $18 \mathrm{~b}$

${ }^{1} \mathrm{H},{ }^{13} \mathrm{C}$ spectra, Compound $18 \mathrm{~b}$ '

${ }^{1} \mathrm{H},{ }^{13} \mathrm{C}$ spectra, Compound $18 \mathrm{c}$

${ }^{1} \mathrm{H},{ }^{13} \mathrm{C}$ spectra, Compound $18 \mathrm{~d}$

${ }^{1} \mathrm{H},{ }^{13} \mathrm{C}$ spectra, Compound $19 \mathrm{a}$

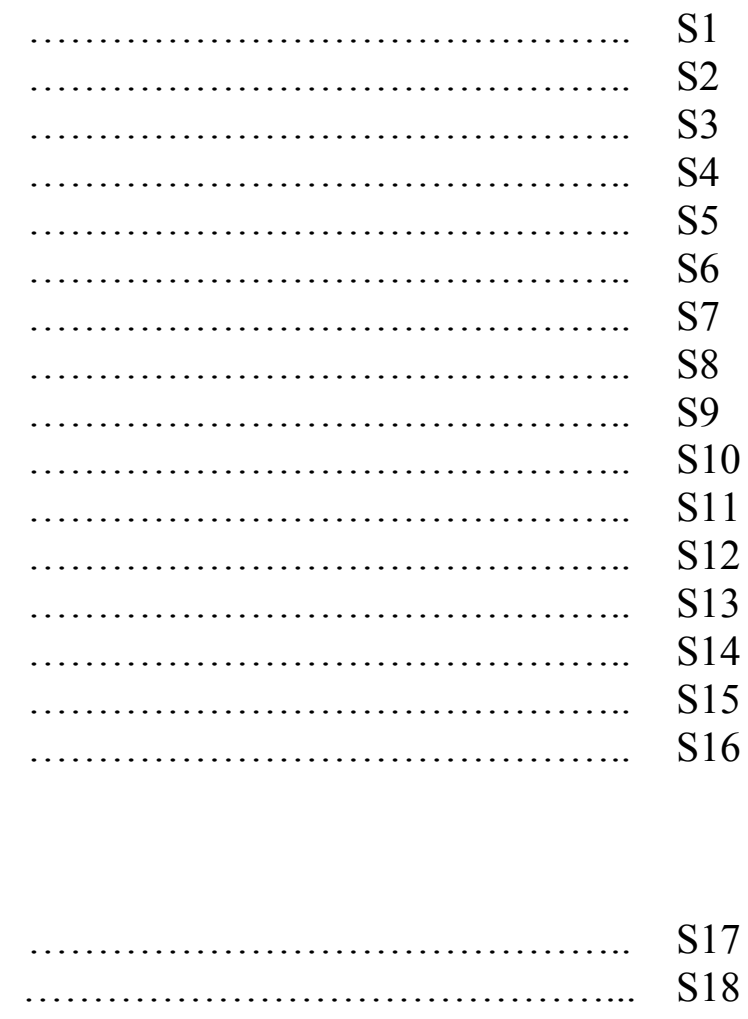

${ }^{1} \mathrm{H},{ }^{13} \mathrm{C}$ spectra, Compound $19 \mathrm{~b}$

${ }^{1} \mathrm{H},{ }^{13} \mathrm{C}$ spectra, Compound $19 \mathrm{~b}$ '

${ }^{1} \mathrm{H},{ }^{13} \mathrm{C}$ spectra, Compound $19 \mathrm{c}$

COSY spectra 19c

Selected multiplets from the ${ }^{1} \mathrm{H}$ -

NMR spectrum of 19c, showing

axial coupling constants for

angular hydrogens

${ }^{1} \mathrm{H},{ }^{13} \mathrm{C}$ spectra, Compound $19 \mathrm{~d}$

General Procedures. All reactions were performed under argon atmosphere, in freshly distilled (under nitrogen) solvents. Unless otherwise noted reagents used are commercially available. Analytical thin-layer chromatography was performed on aluminum plates precoated with silica gel. Visualization was done either with UV light or with phosphomolybdic acid (solution in EtOH). Flash chromatography was performed with hexanes-ethyl acetate mixtures on silica gel with mesh 230-450, under nitrogen pressure. NMR spectra were recorded on $200 \mathrm{MHz}$ or $600 \mathrm{MHz}$ spectrometers. Mass spectra were recorded in-house. The melting points are uncorrected. 
$1 b$

$(\mathrm{OC})_{3} \mathrm{Fe}$ זרan
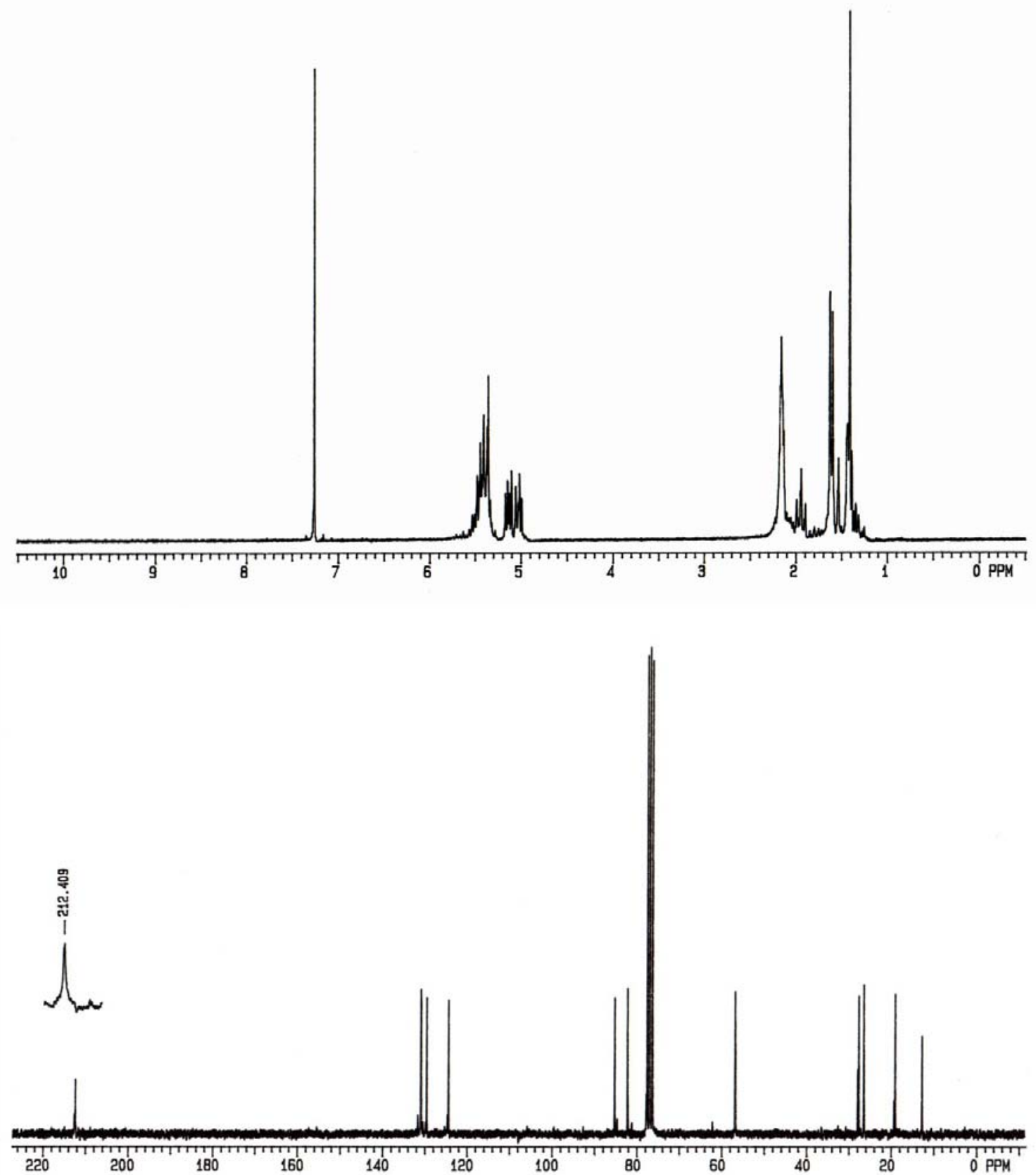
$17 a$

$(\mathrm{OC})_{3} \mathrm{Fe}$
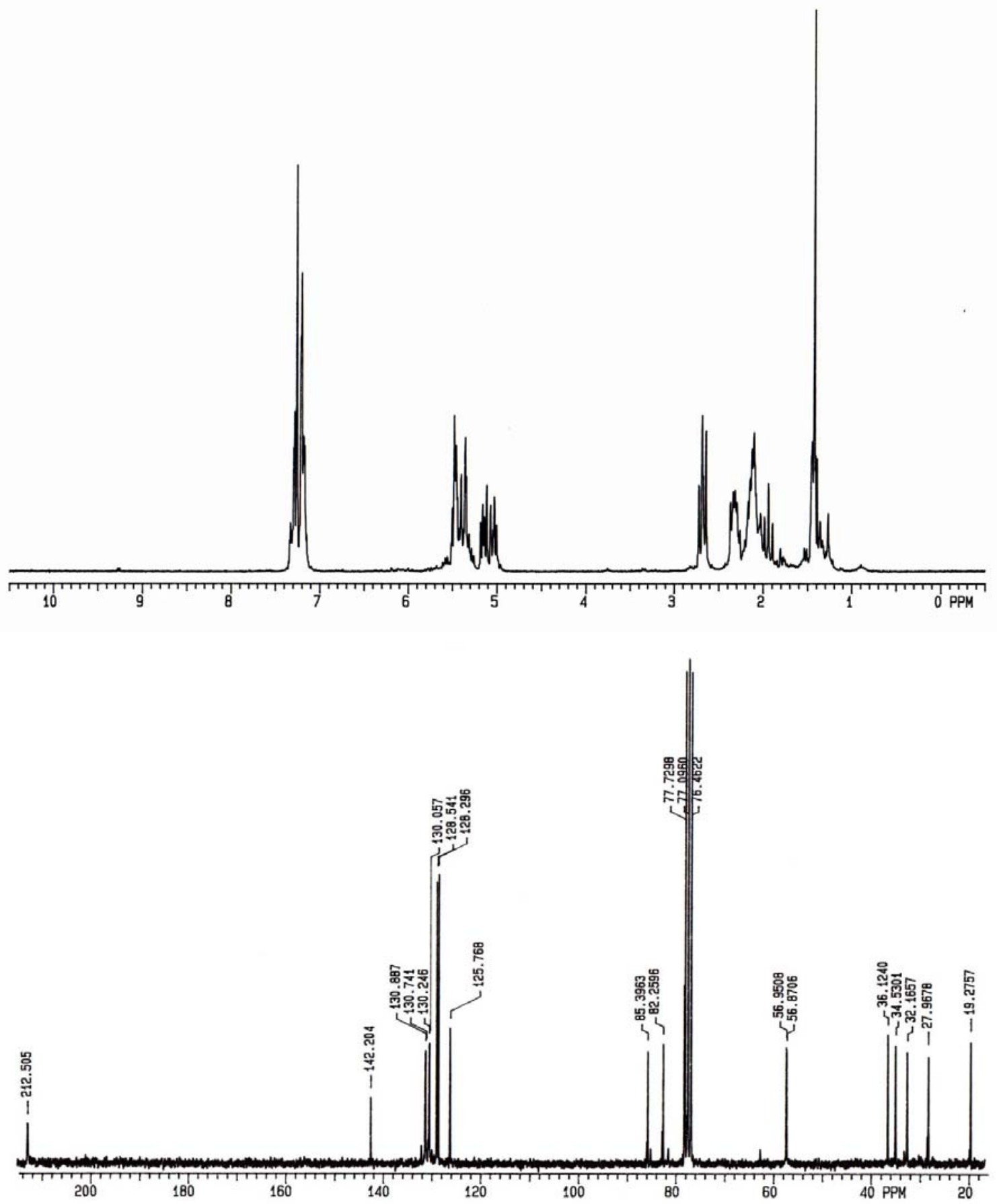
17b
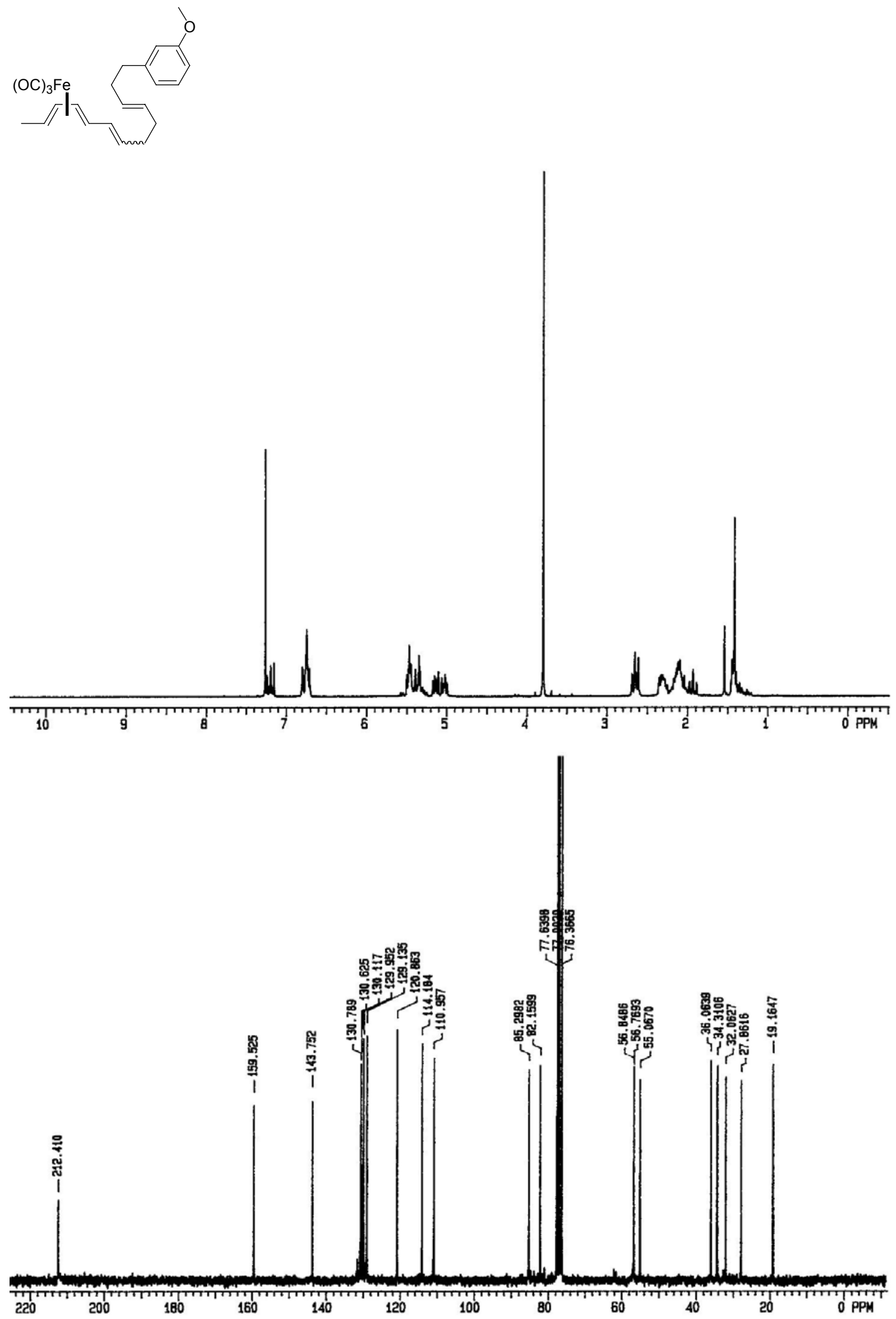
17c

$(\mathrm{OC})_{3} \mathrm{Fe}$
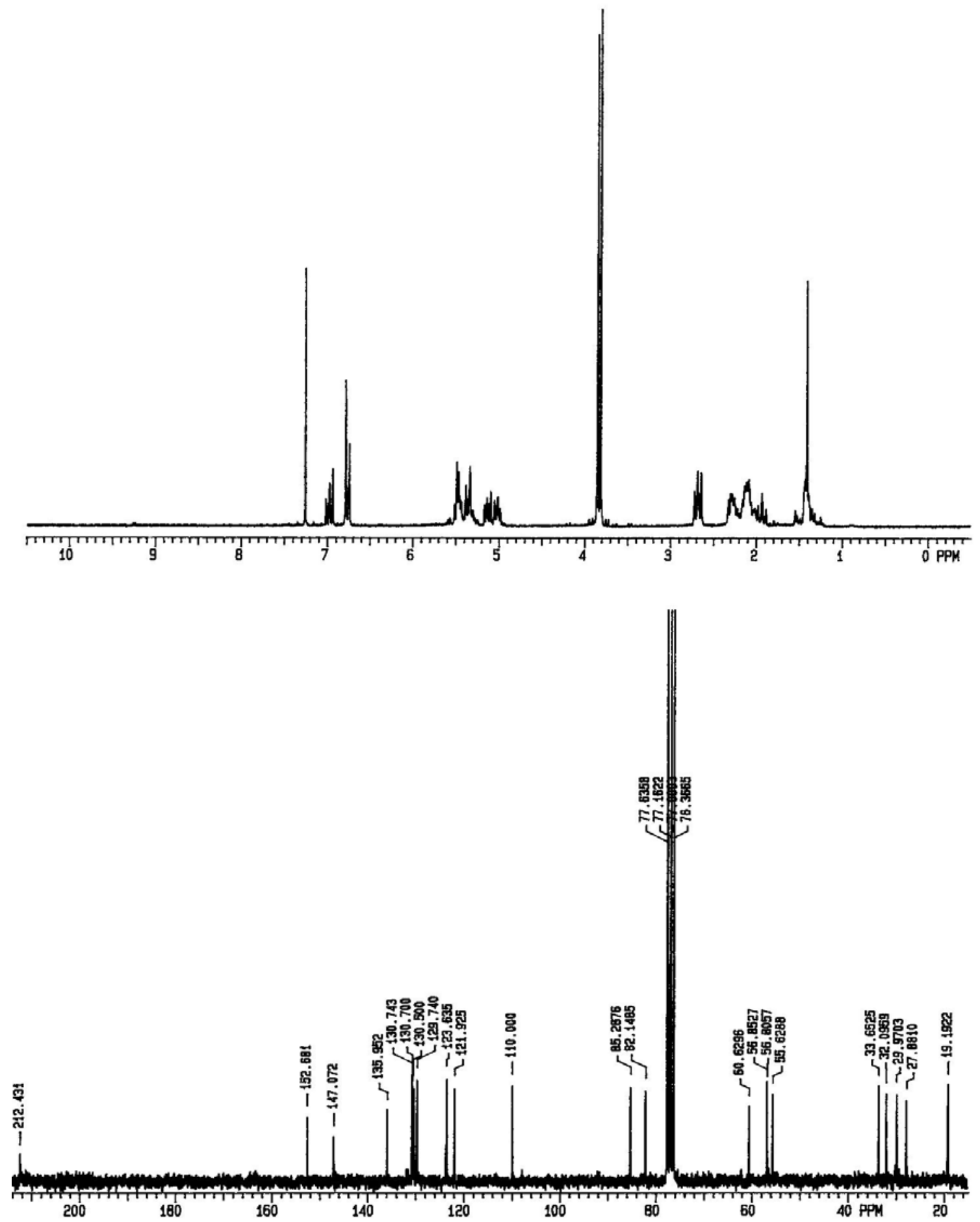


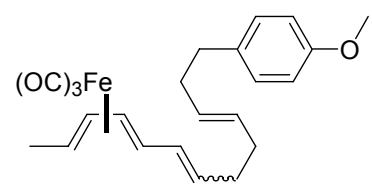
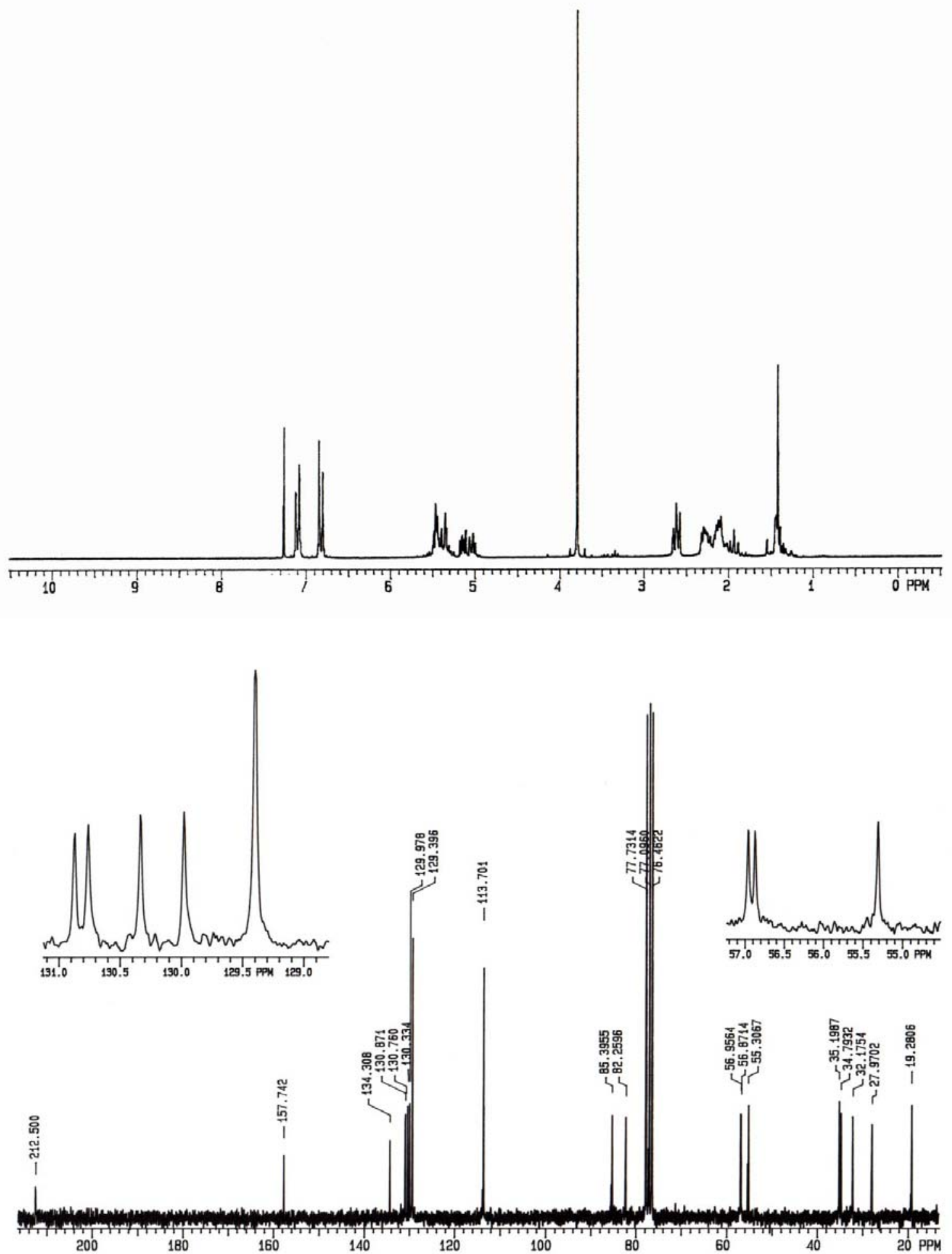
$18 a$

$(\mathrm{OC})_{3} \mathrm{Fe}$
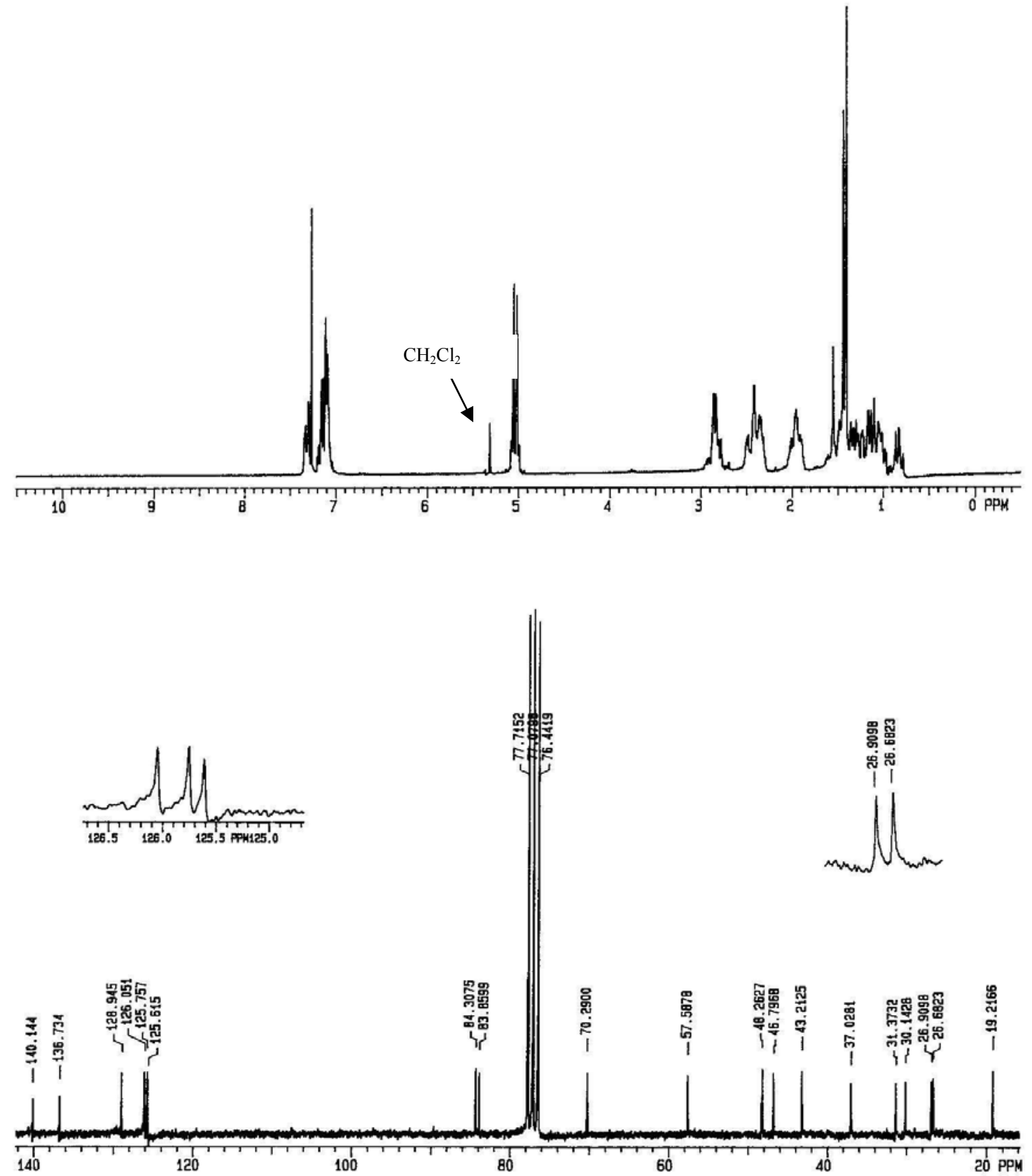
$18 b$
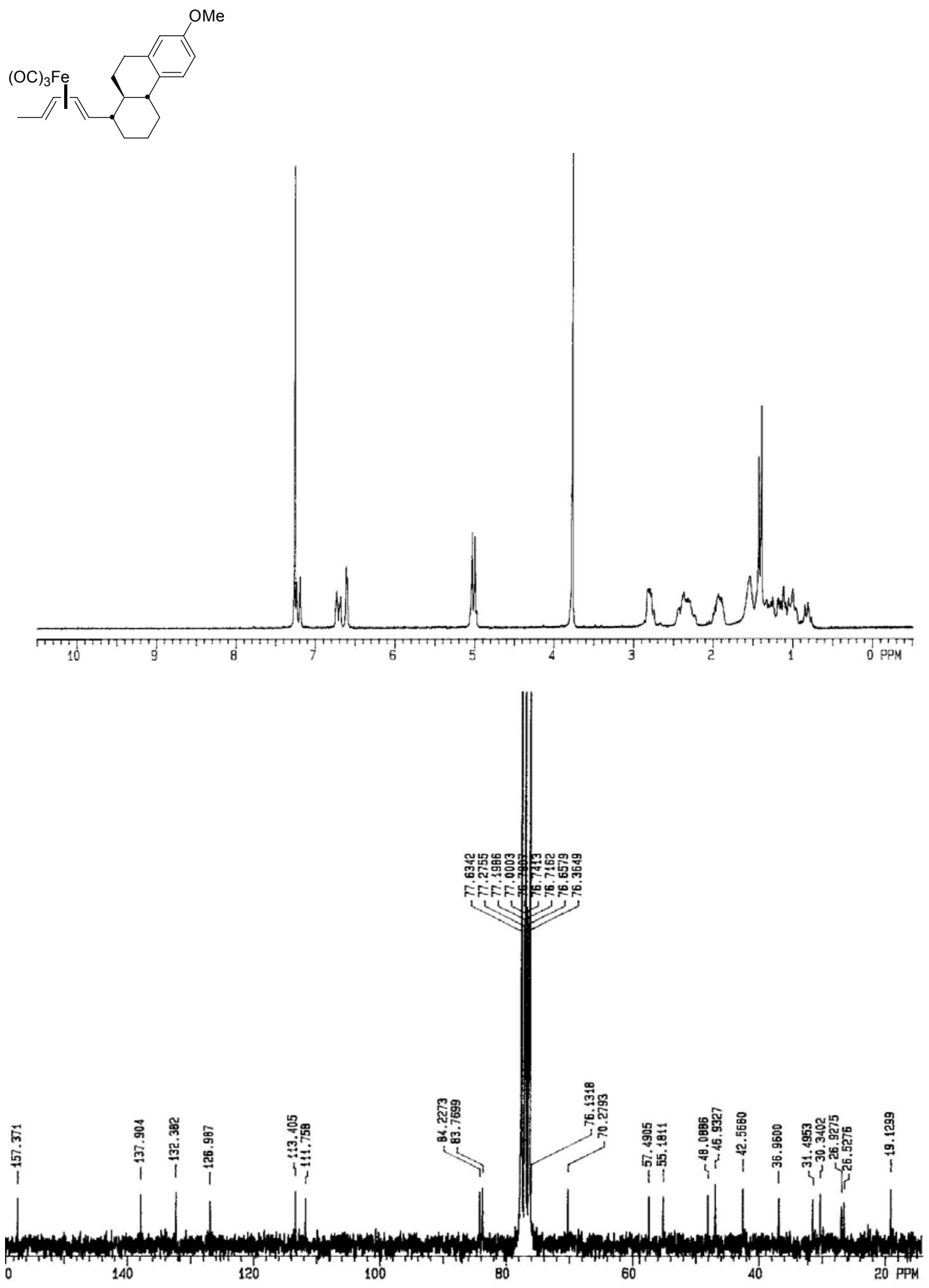
$(\mathrm{OC})_{3} \mathrm{Fe}<\mathrm{OMe}$
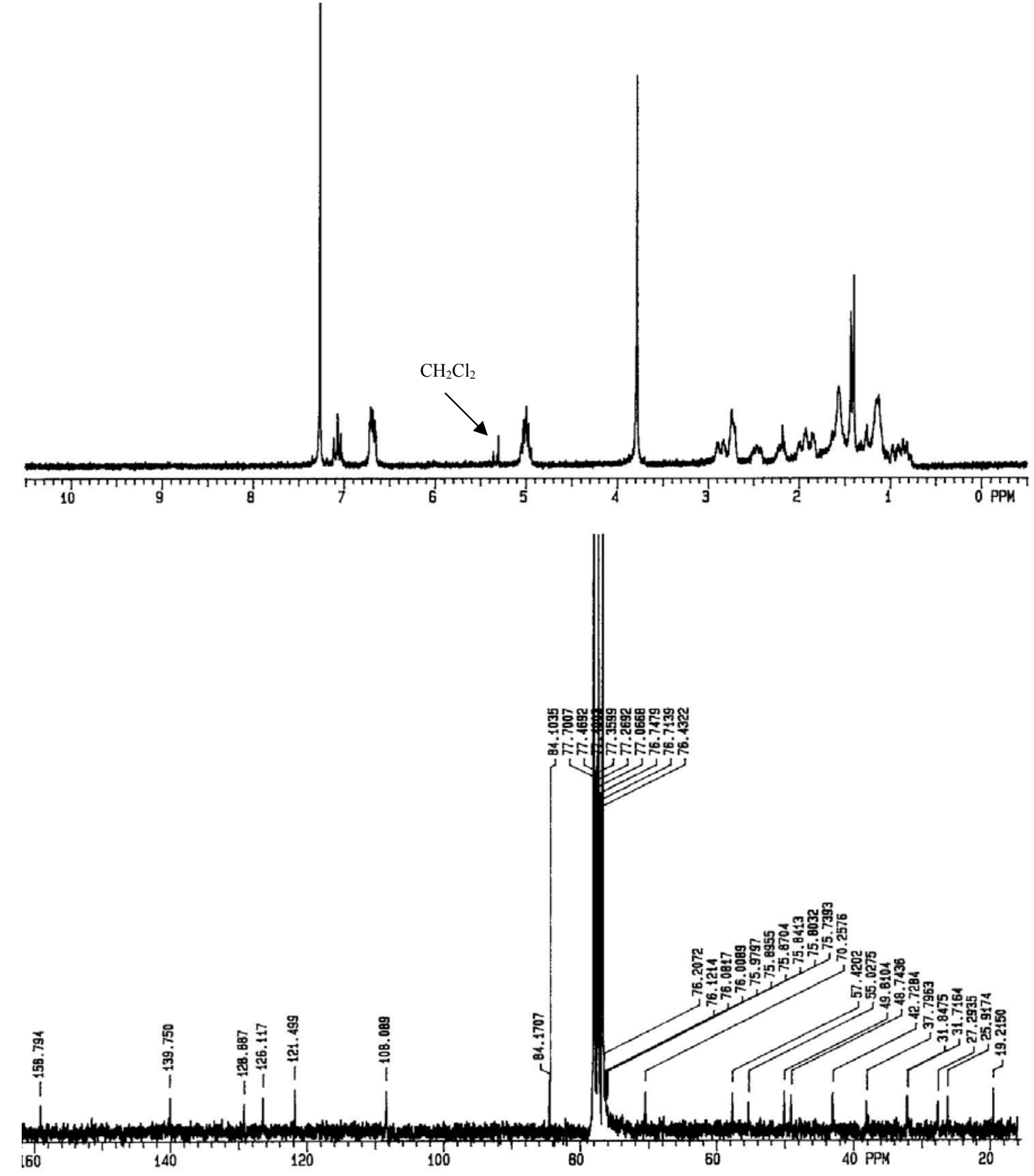
$18 c$

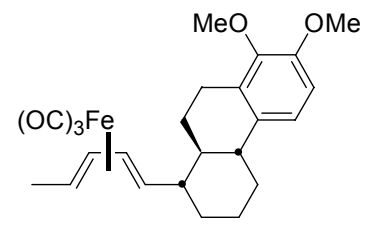

FULL spectrum

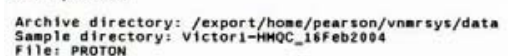

Pulse sequence: szpul
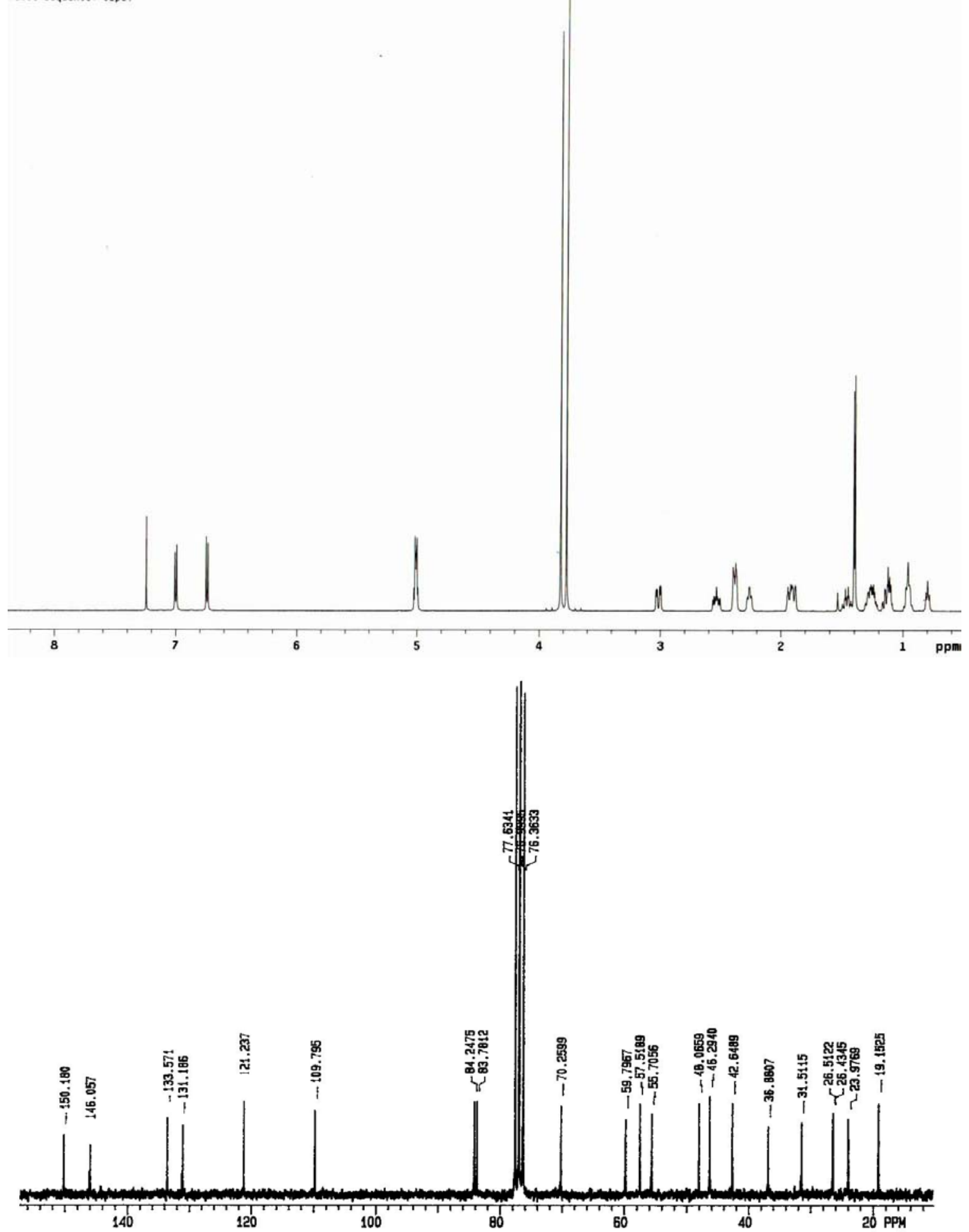
18d

$(\mathrm{OC})_{3} \mathrm{Fe}$
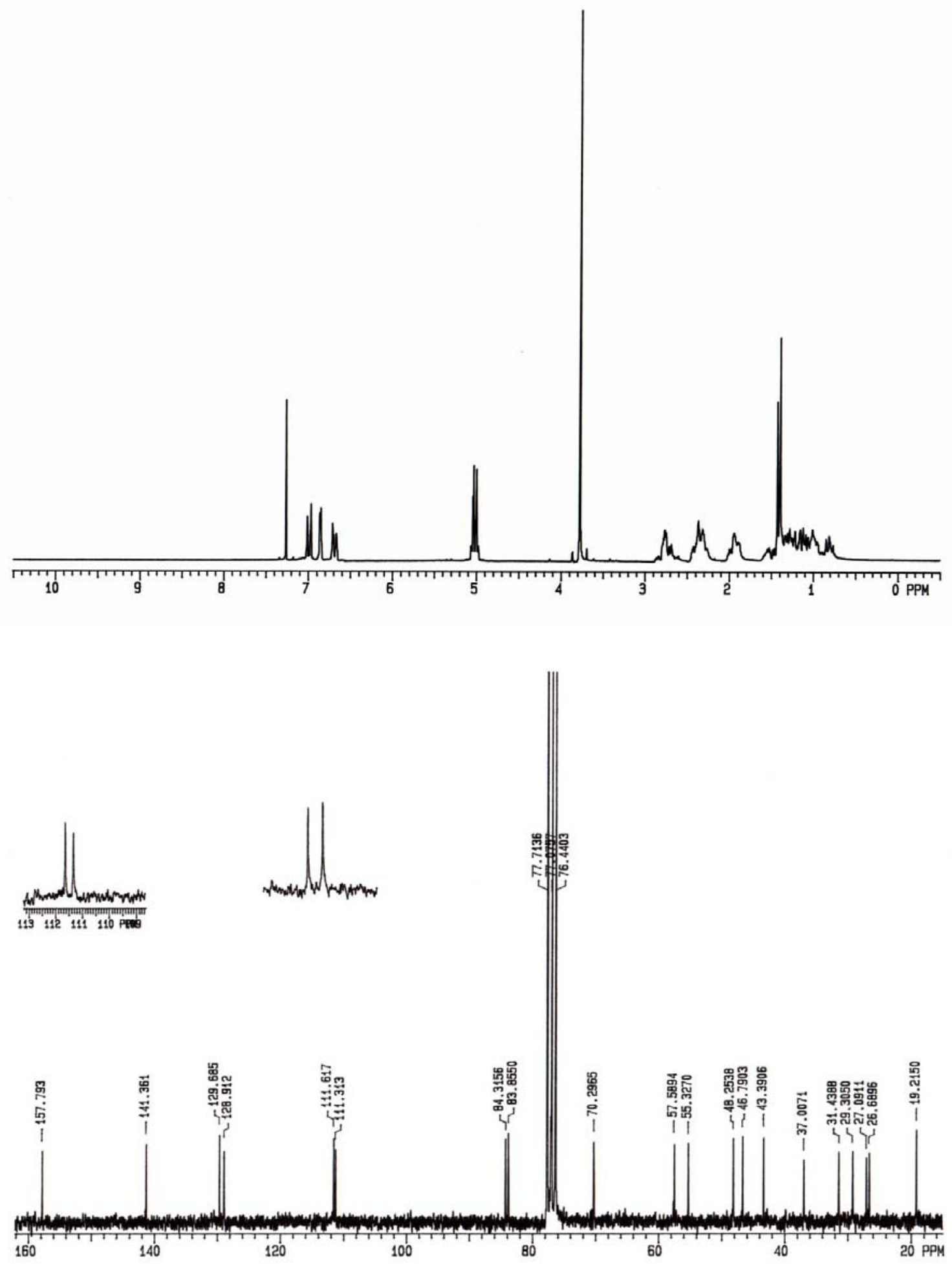
$19 a$

(O)
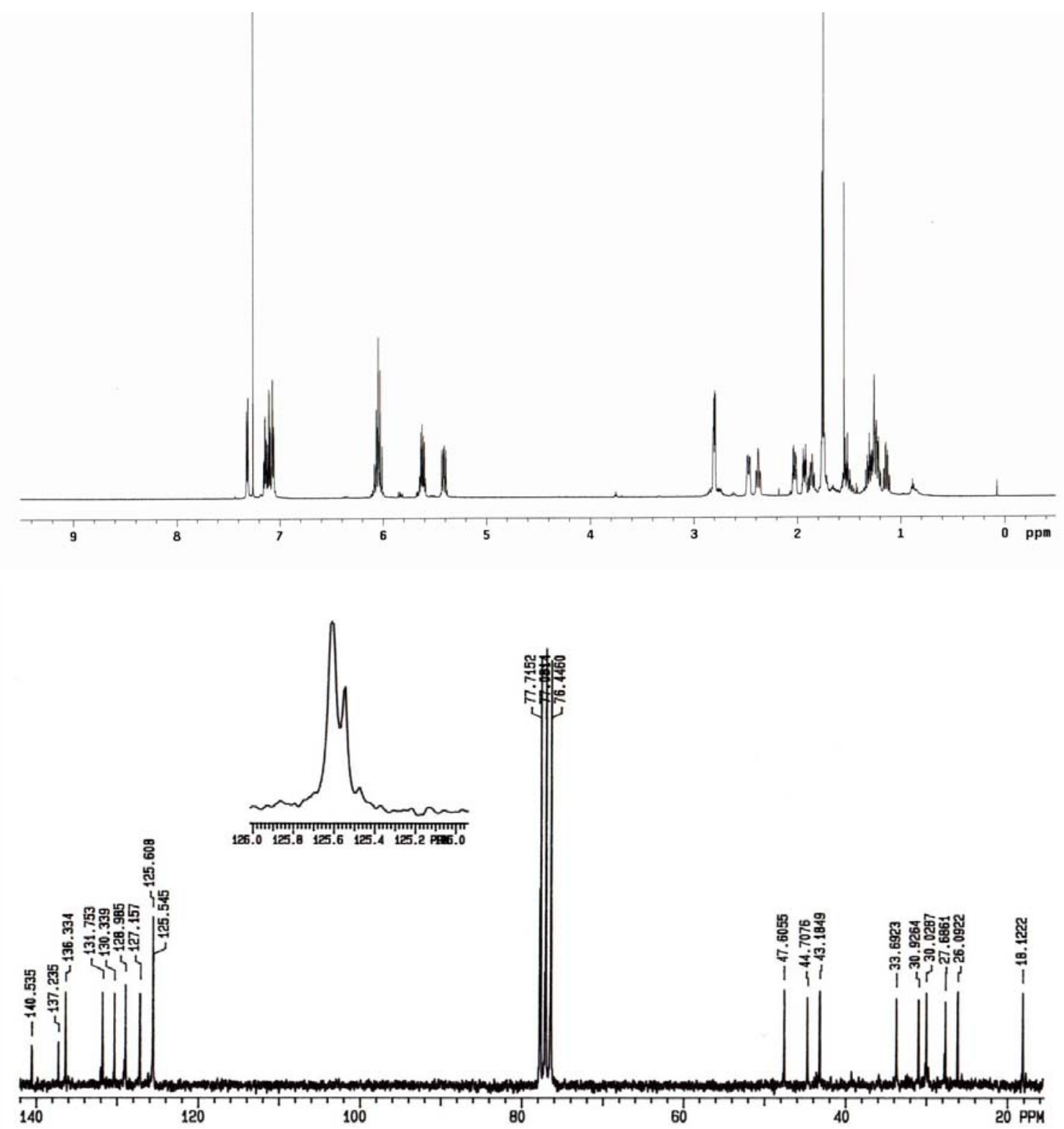
$19 b$

OMe
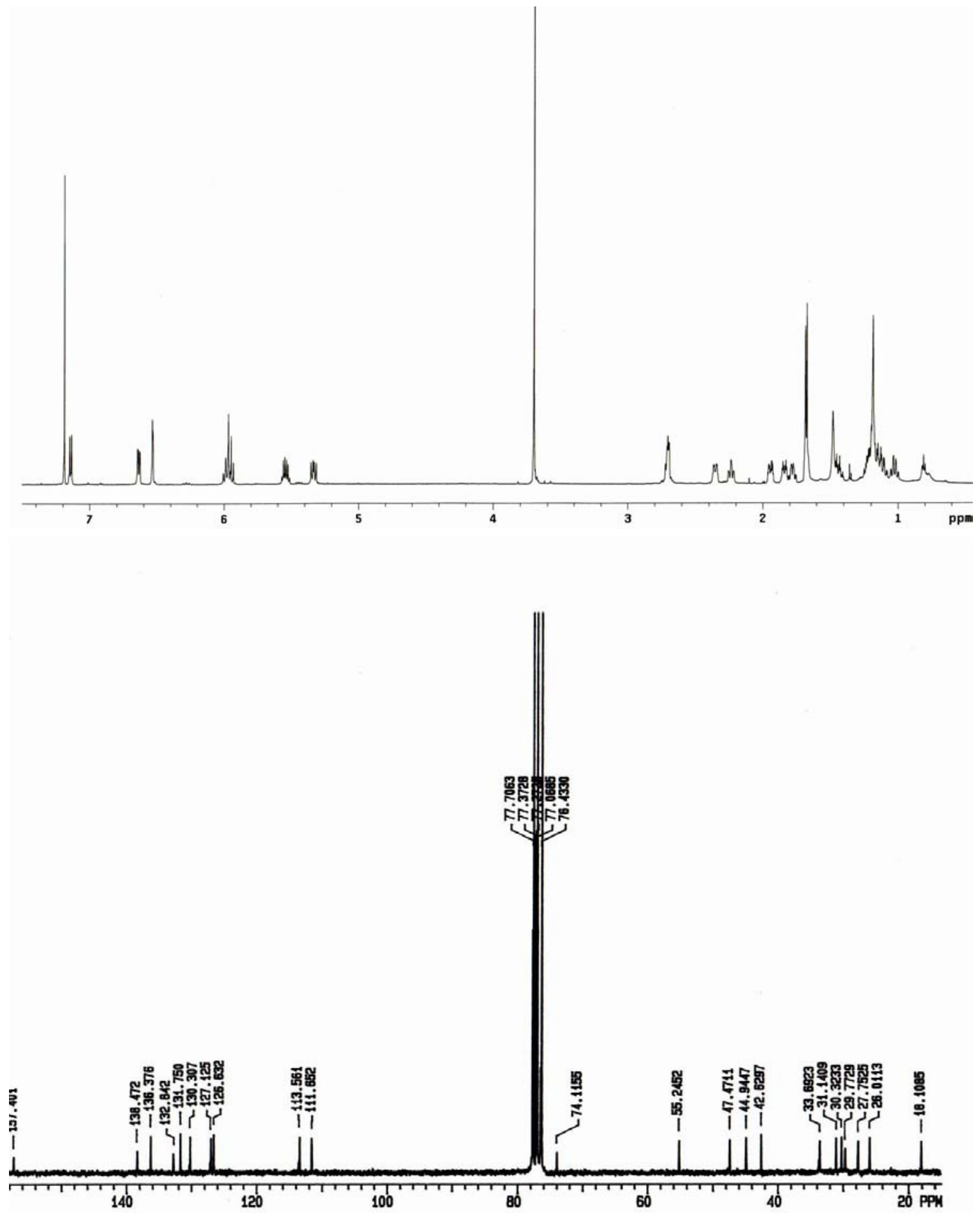
19b'

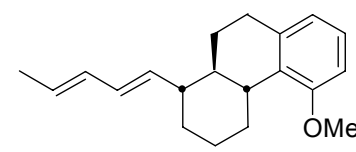

Pulse sequence: s2pul

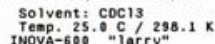

Relax. delayy 1.000 sec

Acg. ${ }^{2}$ ine 3.500 sec

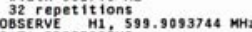

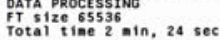
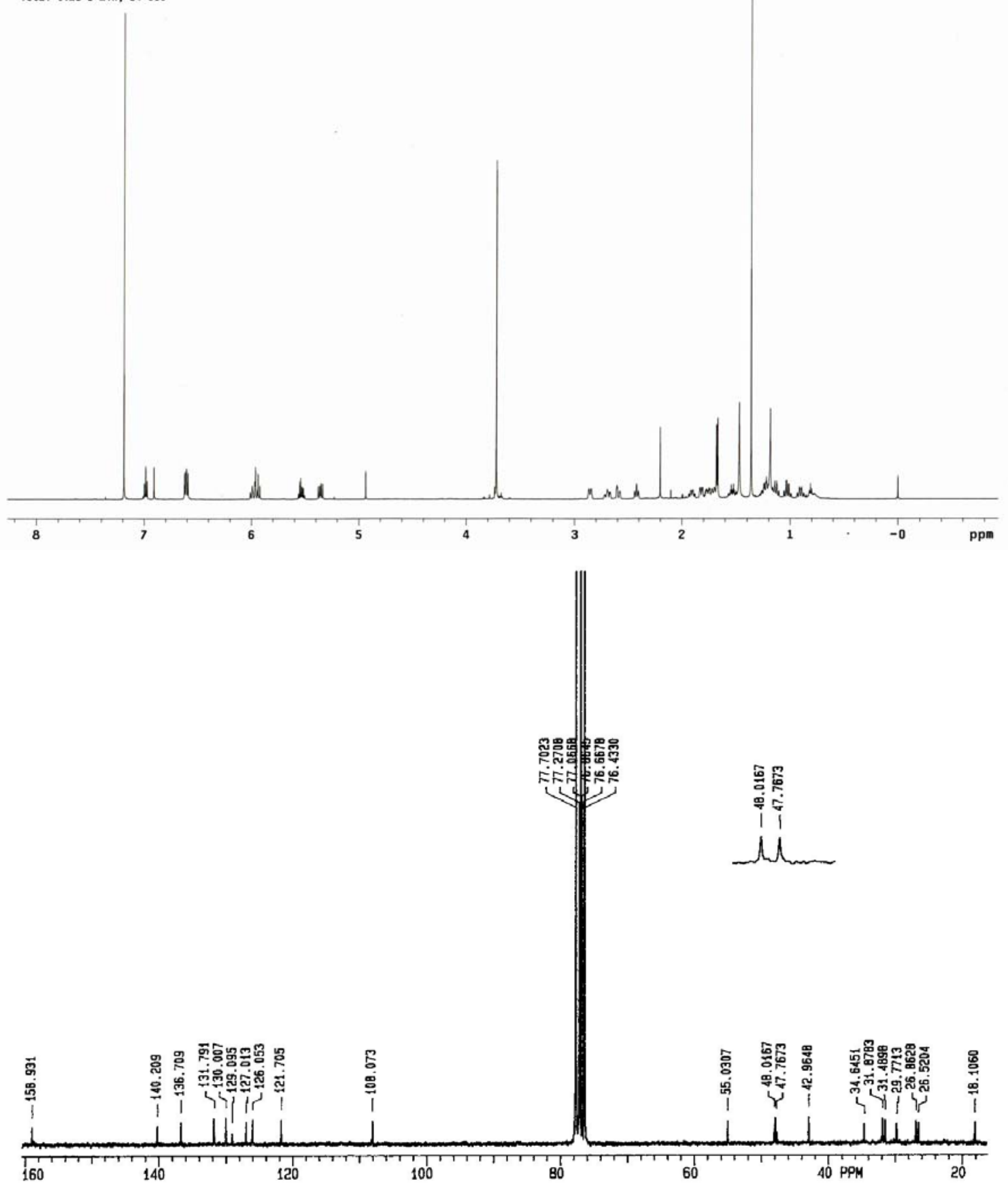
19c

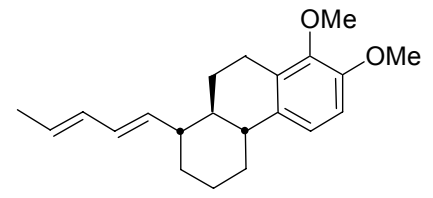

Relax. delay $1.000 \mathrm{sec}$
Pulse

Act. tis.

Gidh $5501.3 \mathrm{~Hz}$

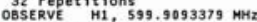

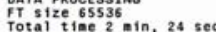
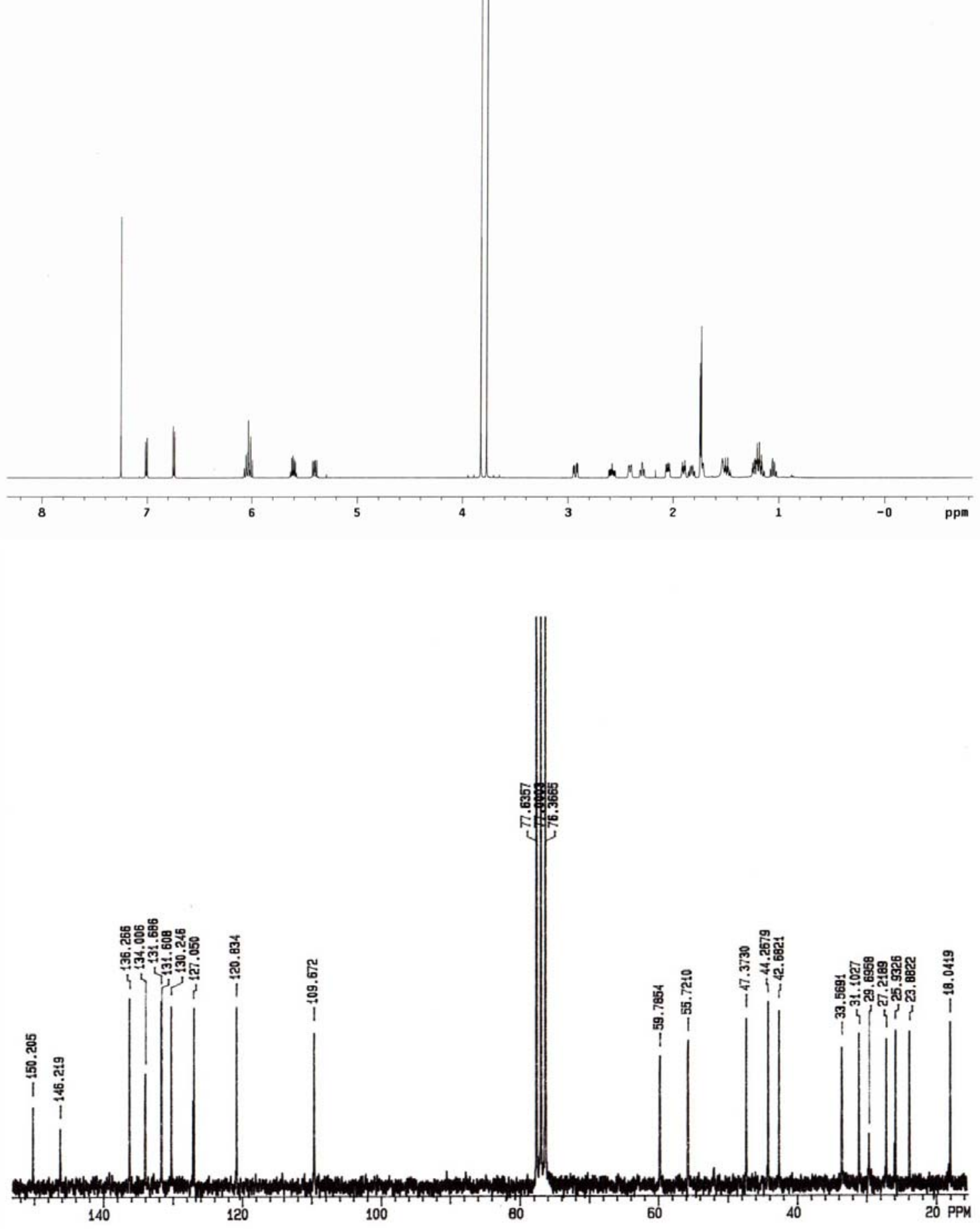


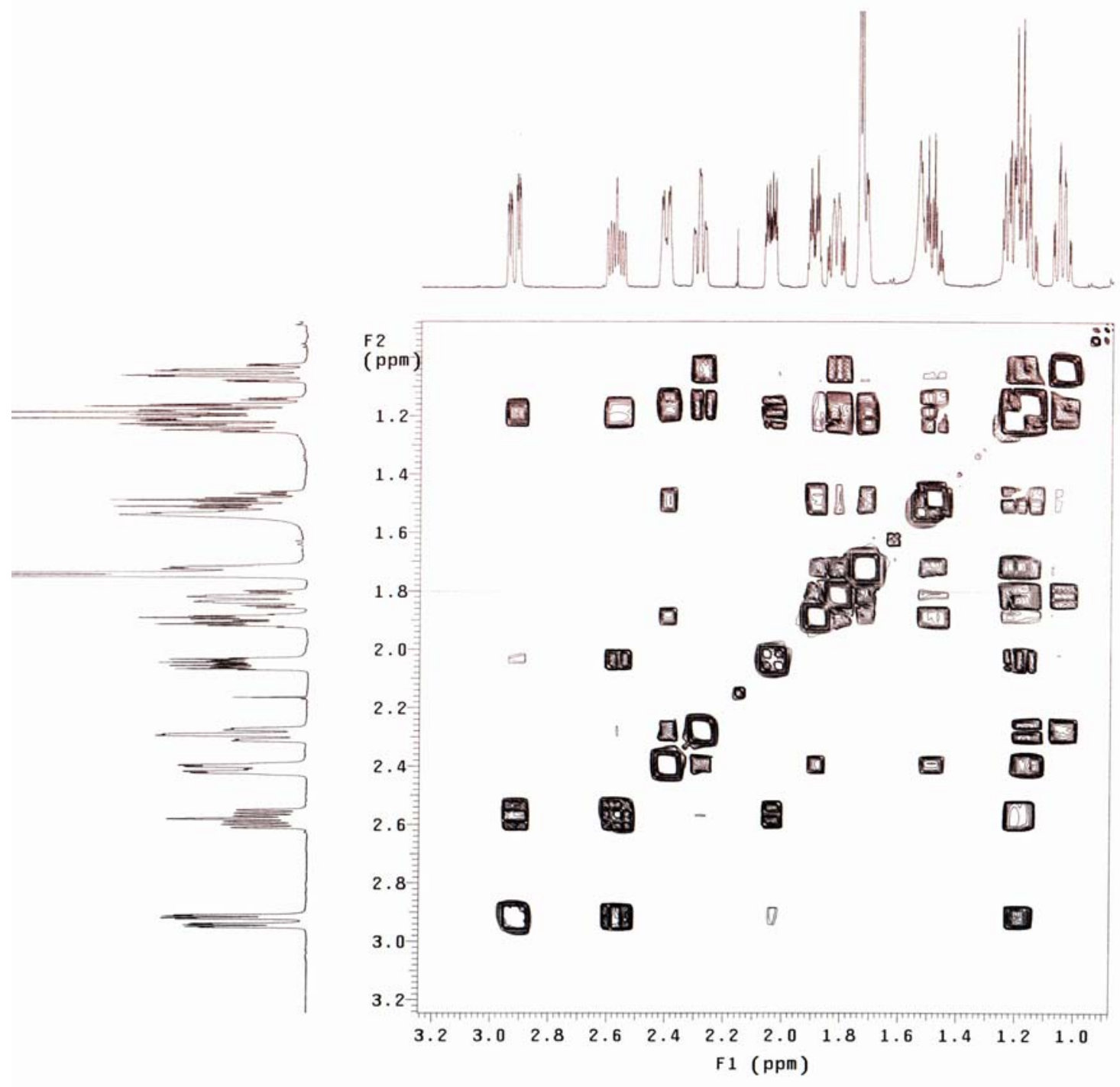


19c - Selected multiplets from the ${ }^{1}$ H-NMR spectrum, showing axial coupling constants for angular hydrogens.

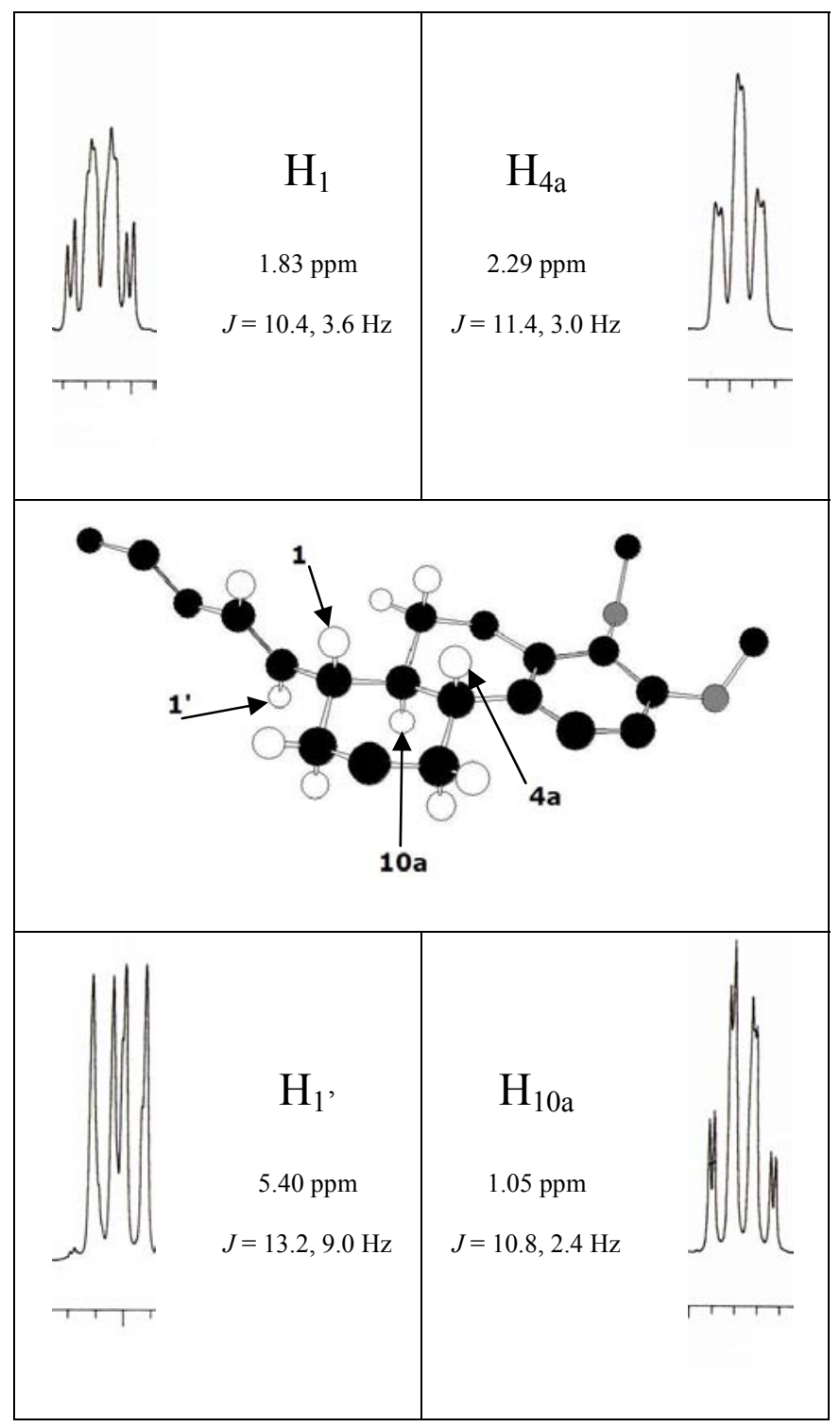


19d

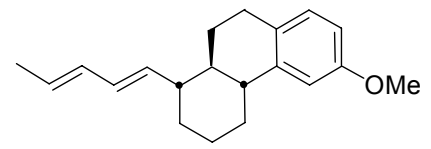

FULL spectrun

Archive directory: / fexport /hoon/pears son/vnarsys/data

Pulse Sequence: szpul
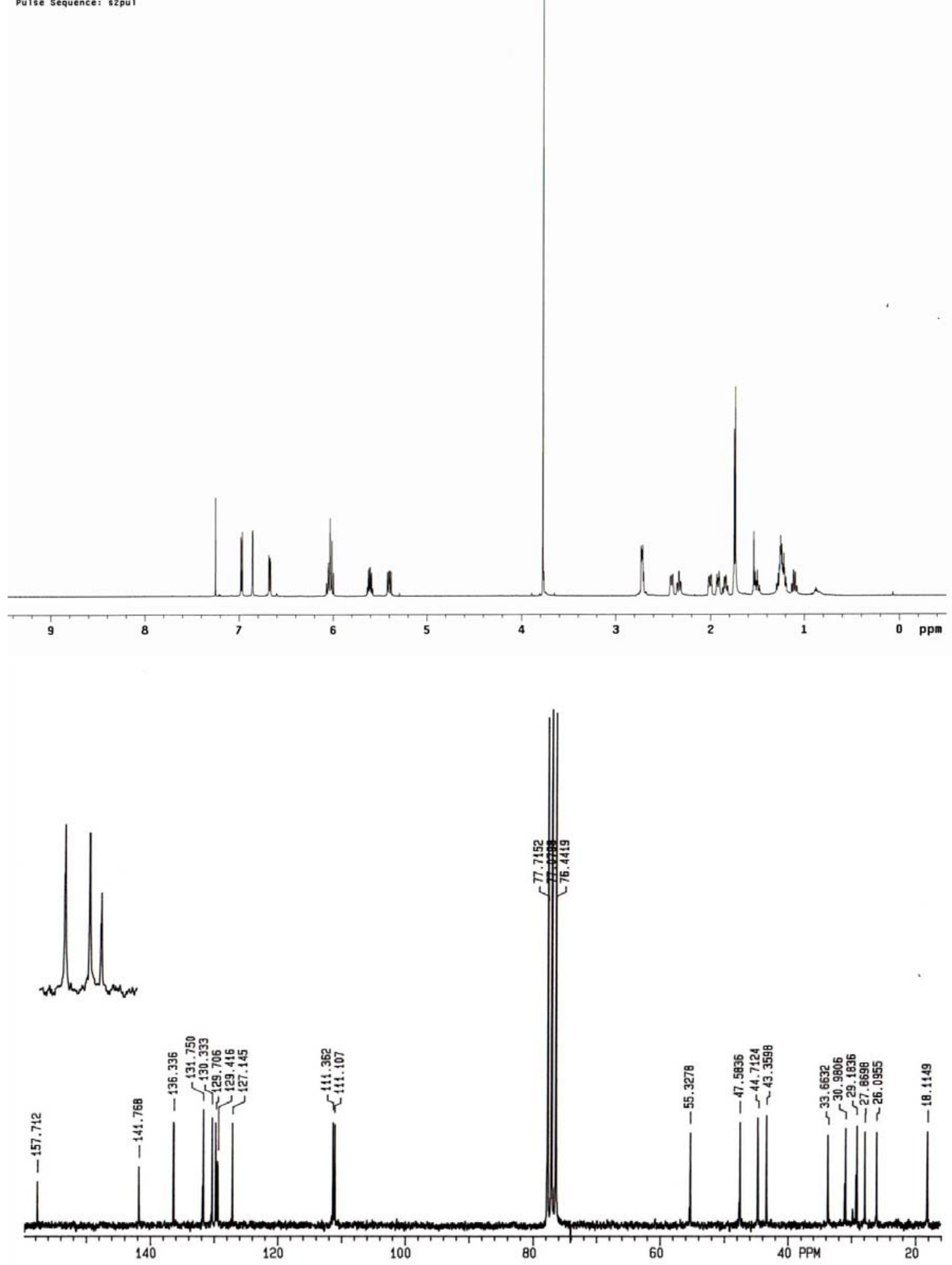